\title{
Coblation annuloplasty in cervical discogenic pain without radiculopathy
}

\author{
Liangliang He, Jiaxiang Ni, Baishan Wu, Jianing Yue, Guoqing Cao, Yuna Guo, Liqiang Yang \\ Department of Pain Management, Xuanwu Hospital, Capital Medical University, Beijing, China \\ Videosurgery Miniinv 2020; 15 (2): 305-312 \\ DOI: https://doi.org/10.5114/wiitm.2019.89394
}

\begin{abstract}
Introduction: The nerve fibers innervating the annulus fibrosus are the major origin of degeneration-associated discogenic pain. Coblation is a tissue-dissociating technique in which the nerve fibers in the degenerative disc tissue are ablated. We hypothesized that coblation annuloplasty would be an effective maneuver for cervical discogenic pain without radiculopathy.

Aim: To observe the therapeutic efficacy of coblation annuloplasty in patients with cervical discogenic pain without radiculopathy.

Material and methods: Forty patients diagnosed with cervical discogenic pain without radiculopathy were screened for coblation annuloplasty therapy. The patient-rated visual analog scale (VAS) score for pain, significant pain relief rate, and Modified MacNab pain-relieving effect were adopted to evaluate the therapeutic effect within a 1-year follow-up period.

Results: Thirty-three patients eventually completed the study. The average pain duration was $4.6 \pm 1.6$ years (range: $0.5-8$ years). The mean VAS pain score decreased from preoperative $6.8 \pm 0.9$ to postoperative $2.5 \pm 1.3(p<0.01)$. For all participants, the immediate pain relief rate was 78.7\% (26/33), which continued to postoperative 6 months. One year later, $22(66.6 \%)$ subjects reported that their pain was significantly alleviated. According to the Modified MacNab criteria, 63.6-82.1\% considered the effect of surgery for their pain therapy as "excellent" during the 1-year follow-up period. No significant complications such as hemorrhage, paresthesia, or infection were observed. Conclusions: This study is the first to demonstrate that coblation annuloplasty is an effective intervention providing significant alleviation of neck pain from cervical discogenic injury without radiculopathy.
\end{abstract}

Key words: coblation, annuloplasty, cervical, discogenic pain.

\section{Introduction}

Cervical discogenic pain is a complex pain condition in which algesia and paresthesia present in the neck area with or without radiculopathy [1]. Its precise diagnostic criteria are not yet explicitly defined due to the presence of many coexistent and overlapping radicular and somatic symptoms [2]. An estimated 2 billion individuals worldwide are afflicted with various types of pain [3]; of them, $24-80 \%$ originate in the spine [4]. The prevalence of neck pain was around $15 \%$ in individuals who reported grade II-IV pain intensity [5]; pain is the fourth leading cause of disability, with an annual prevalence exceeding $30 \%$ [6]. Most of these cervical pain-afflicted patients received only conservative therapies, and the rest were given limited interventional treatments [7, 8]; however, the therapeutic efficacy of these interventions showed huge variance [9], from which some researchers created a multimod-

\footnotetext{
Address for correspondence

Liqiang Yang, Department of Pain Management, Xuanwu Hospital, Capital Medical University, Beijing, China,

e-mail: cnjiaxiangni@163.com
} 
al program for achieving effective pain relief [10]. It is of practical importance to determine an effective method with consistent therapeutic efficacy at controlling cervical discogenic pain (CDP).

Compared to interventions used for lumbar back pain, neck pain treatments are challenging due to surgery-related anatomic limitations and the risk of technique-associated thermal injury to the cervical nerve root $[2,11]$. In addition, more than two-thirds of cases of discogenic pain are not related to nerve root compression; rather, they are only derived from the inflammatory evocation of the nerve fibers distributed in the outer one-third of the annulus fibrosus [12]. These innervating fibers branch from the interconnected sinuvertebral and vertebral nerves that convey nociceptive signals up into the cerebral pain centers $[12,13]$. Thus, the conventional therapeutic maneuvers cannot produce effective pain relief in cases of CDP because they will not attenuate the firings of the overactivated discal nerve fibers. Meanwhile, anti-inflammatory medication injections concentrating on the sole discogenic pain condition are also not the therapy of choice because their effectiveness is too short-term [9]. Moreover, thermal-associated annuloplasty including intradiscal thermal annuloplasty (IDET), radiofrequency annuloplasty (RFA), and intradiscal biacuplasty (IDB) all carry a high risk of injury to the cervical nerve roots due to their specific anatomic locations. Therefore, it is critical to identify an intervention that possesses clinical implications if it can produce a long-lasting therapeutic effect on CDP without increasing nerve root injury risk over currently available methods.

Coblation, a tissue dissociating technique independent of a heat-driven process, is a broadly applied medical maneuver with a central temperature of $40-70^{\circ} \mathrm{C}$ that produces minimal thermal damage to the surrounding healthy tissue since its radiofrequency current does not pass directly through the tissue [14]. A thermal mapping study using a porcine model demonstrated that the temperature surrounding the coblation probe decreased significantly in a distance-dependent manner, i.e. the temperatures decreased from $40^{\circ} \mathrm{C}$ to $20^{\circ} \mathrm{C}$ to $0^{\circ} \mathrm{C}$ when the radius to the probe tip extended from 0 to 1 to $5 \mathrm{~mm}$ [15]; a further study showed that coblation is a safe method of ablating vertebral lesions without harboring a danger of thermal injury to the spinal cord or spinal nerves [16]. Although coblation nucleoplasty has been a preferred method to treat cervical radicular pain from severe compression of the herniated nucleus pulposus to the spinal nerves for the past decade $[17,18]$, its therapeutic efficacy for discogenic pain is insufficient [19]. We proposed that annuloplasty combined with coblation would be an effective therapeutic choice for cervical discogenic pain through which both intrinsic nerve fibers innervate the outer layers of the annulus fibrosus [12] and the possibly newly ingrown nerves into the central layers of intervertebral disc are derived from the chronic degenerative process [20].

\section{Aim}

In 2017, we initiated a pilot study to observe the therapeutic efficacy of coblation annuloplasty in patients with cervical discogenic pain without radiculopathy in China.

\section{Material and methods \\ Objects}

Ethical approval was obtained from our hospital's Institutional Ethics Examining Committee of Human Research prior to patient recruitment. All participants provided informed written consent and were provided a full explanation of the coblation annuloplasty and its schematic procedures, benefits, and potential complications. Recruitment for coblation annuloplasty for cervical discogenic pain therapy occurred between August 2017 and January 2018. The data were collected at a university-affiliated tertiary teaching hospital in China.

All patients enrolled in this study were diagnosed with sole cervical discogenic pain without radiculopathy. Therefore, all participants met the following diagnostic criteria: (i) neck pain without radicular pain; (ii) visual analog scale (VAS) score pain intensity $\geq 4$; (iii) pain duration $\geq 3$ months; and (iv) magnetic resonance imaging (MRI) findings showing a herniated disc $\leq 3 \mathrm{~mm}$ affecting less than $1 / 3$ of the central spinal canal.

In addition to the above diagnostic criteria, all included patients also met the criteria below for the coblation annuloplasty procedure: (i) either sex; (ii) age $>18$ years of age and full comprehension of the surgical procedures and mature physical development; (iii) no neurological deficits, such as loss of sensory, motor, or reflex; and (iv) failure of conser- 
vative management including medication, physical therapy, and fluoroscopically directed injections.

Patients with any of the following conditions were excluded: (i) coagulopathy; (ii) uncontrolled psychological or psychiatric disorders; (iii) disc herniation with sequestration, infection, or spinal instability; (iv) spinal fracture; (v) spinal tumor; (vi) advanced spondylosis resulting in osseous foraminal stenosis or disc space collapse; (vii) surgical history on the spine at the same cervical level; (viii) allergy to local anesthetics; (ix) unwillingness to participate in the study; (x) current or past history of drug addiction that would affect cognition and judgment; (xi) use of medications such as analgesics, antipsychotics, or hypnotics; or (xii) any diagnosed congenital or idiopathic or acquired disease of the nervous system.

This pilot prospective observational study aimed to obtain preliminary data concerning the therapeutic efficacy of coblation annuloplasty for patients with cervical discogenic pain without radiculopathy.

\section{Procedures}

None of the patients received any sedative or anticholinergic medications prior to the surgical procedures. After entering the operating room, the patient was placed in the supine position with a $10-\mathrm{cm}$ thick cushion placed under the shoulder to slightly extend the neck. Oxygen $3 \mathrm{l} / \mathrm{min}$ was applied to all patients via a face mask throughout the procedure. Etimicin $1.0 \mathrm{~g}$ was administered prophylactically 30 min prior to surgery. Fentanyl $50 \mu \mathrm{g}$ was injected intravenously to provide analgesia, but no other sedatives were used to keep the patient conscious and able to respond to nerve root stimulation during surgery. Heart and respiratory rate, three-lead electrocardiography, and fingertip digital oximetry were monitored throughout the process.

Patients were placed in the supine position on the operation table and a $10-\mathrm{cm}$ cushion was placed under the shoulder to keep the neck slightly hyperextended. The patient's vital signs were monitored. After sterilization of the surgical area, $5 \mathrm{ml}$ of lidocaine $10 \mathrm{mg} / \mathrm{ml}$ without adjuvants was used for local analgesia and patients received a puncture in a left or right anterior approach under fluoroscopic guidance on anteroposterior (AP) and lateral views.

First, the puncture angle was confirmed under fluoroscopic guidance on the AP and lateral views. Second, an 18-gauge 8-cm introducer needle was advanced via a left or right anterior approach to the target disc. During the puncture, the introducer needle was inserted slowly and stopped immediately once a twitch movement was observed or paresthesia was reported by the patient on their corresponding upper limb. Once the introducer needle entered into the target disc, it was advanced slowly until the tip reached the opposite posterior annulus-nucleus junction. We checked the tip position with fluoroscopy on AP (Photo 1 A) and lateral (Photo 1 B) views. Third, the coblation wand (UNITEC, China America United Technology (Beijing) Co. Ltd., Beijing, China) was inserted into the introducer needle until the its tip was extended approximately $5 \mathrm{~mm}$ beyond the tip of the needle to ensure the active portion of the wand was deployed into the annulus. We checked the wand tip position with fluoroscopy in the AP (Photo $1 \mathrm{C}$ ) and lateral (Photo $1 \mathrm{D}$ ) views. We tested the coagulation with the radiofrequency controller set at $2^{\prime}$ for $0.5-1 \mathrm{~s}$ to ensure that no limb movement or paresthesia occurred. We performed the coblation with the radiofrequency controller set at 2' of intensity to ablate the disc materials by rotating the wand $360^{\circ}$. Each coblation cycle was less than $10 \mathrm{~s}$, and a total of three coblation cycles were performed. We conducted coagulation with the radiofrequency controller set at 2' intensity for 1-2 s to denature the adjacent materials and seal the channel. Once the coblation wand was withdrawn, $2 \mathrm{ml}$ of $0.5 \%$ lidocaine was applied to the introducer needle tract.

Postoperatively, all patients rested in the supine position in bed for at least $48 \mathrm{~h}$. They were discharged home with instructions to avoid strenuous activities and adhere to the following: (1) wear a neck brace at least 1 week when sitting or walking; (2) discontinue wearing the neck brace after no more than 1 month to avoid the potential risk of neck myophagism; (3) avoid long-lasting head lowering; and (4) keep the cervical spine in extension and flexion position for no more than 30-60 min and then relax for at least $10 \mathrm{~min}$.

The primary outcome of the study was the VAS pain score, in which a $100-\mathrm{mm}$ gauge $(0=$ no pain, $100=$ worst pain imaginable) was used. VAS pain scores were recorded at 1 week and 1, 3, 6, and 12 months postoperatively.

The following variables were recorded as the secondary outcomes. Significant pain relief was defined as postoperative pain relief $\geq 50 \%$ compared to preoperative values rated at 1 week and 1, 3, 6, and 12 months after surgery. Functional status was qual- 

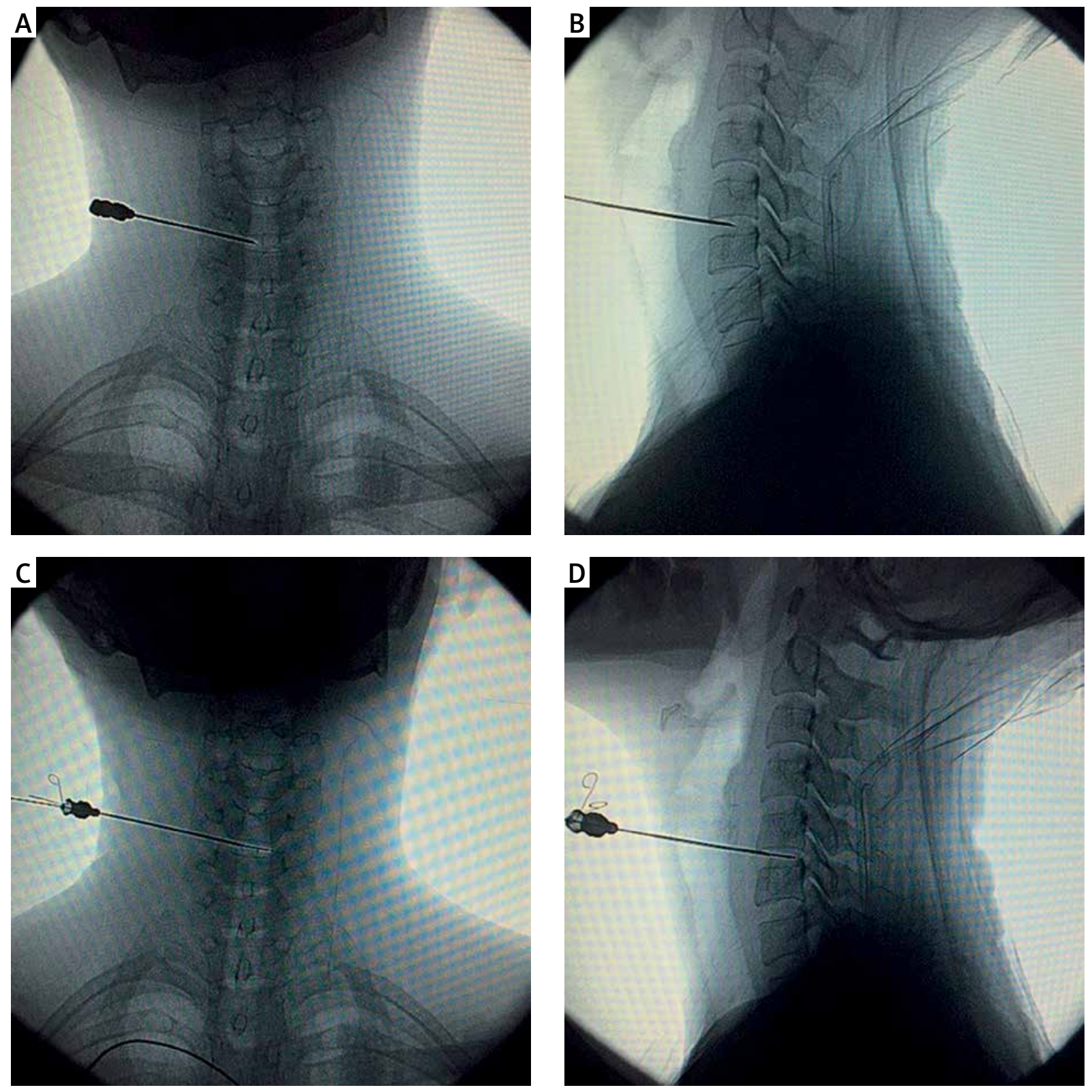

Photo 1. Introducer needle was advanced to the posterior annulus/nucleus junction at C5/6 as seen in the anterior-posterior (AP) view (A) and in the lateral view (B), the tip of coblation wand in the posterior annulus at C5/6 as seen in the AP view (C) and in the lateral view (D)

ified as "excellent", "good", "fair" and "poor" according to the Modified MacNab criteria and recorded at 1, 3, 6, and 12 months postoperatively. Finally, complications such as hemorrhage, paresthesia, and infection were recorded.

\section{Statistical analysis}

Statistical analyses were performed using GraphPad Prism version 5.0 (GraphPad Software,
Inc., San Diego, CA, USA). All values are expressed as mean, SD, interquartile range, or numbers. Oneway analysis of variance was used to compare the repeated measures such as VAS pain scores among different time points. The Wilcoxon sign rank test was adopted to evaluate the situation of significant pain relief and functional status. Statistical significance was accepted at the level of $p<0.05$. 


\section{Results}

A total of 40 patients were recruited; of them, 7 were excluded. Thus, a total of 33 subjects underwent coblation annuloplasty therapy and completed the study. Of the 7 excluded patients, 2 (5\%) withdrew consent after surgery, 2 (5\%) lost contact during the 1-year follow-up period; 1 (2.5\%) had a coagulation problem due to anticoagulation therapy for a previous cardiac stent, 1 (2.5\%) had a surgical history at the same cervical level, and 1 (2.5\%) was taking a hypnotic for sleeping. Table I summarizes the demographic, background characteristics, and baseline vital signs (all were within the physiologic ranges).

The pain characteristics of our patients are shown in Table II. The average pain duration was 4.6 \pm 1.6 years (range: $0.5-8$ years). Eleven $(33.3 \%)$ had C4/5 disc, 21 (63.6\%) had C5/6 disc, and 1 (3\%) had C6/7 disc problems.

The average self-rated preoperative pain intensity was $6.8 \pm 0.9$, and the surgery significantly alleviated pain intensity to $2.5 \pm 1.3$; this alleviation

Table I. Demographic characteristics

\begin{tabular}{|lc|}
\hline $\begin{array}{l}\text { Parameter } \\
\text { Gender: }\end{array}$ & $\begin{array}{c}N(\%) \text { or mean } \pm \text { SD, } \\
\text { or (min.-max.) }\end{array}$ \\
\hline Male & $15(45.5)$ \\
\hline Female & $18(54.5)$ \\
\hline Age [years] & $49 \pm 7(31-61)$ \\
\hline Duration of pain [years] & $4.6 \pm 1.6(0.5-8)$ \\
\hline \begin{tabular}{l} 
Distribution of pain: \\
\hline Neck base
\end{tabular} & $5(15.2)$ \\
\hline $\begin{array}{l}\text { Neck base } \\
\text { Shoulder top }\end{array}$ & $10(30.3)$ \\
\hline $\begin{array}{l}\text { Neck base } \\
\text { Shoulder top }\end{array}$ & $11(33.3)$ \\
\hline $\begin{array}{l}\text { Upper back } \\
\text { Shoulder top }\end{array}$ & $21(63.6)$ \\
\hline $\begin{array}{l}\text { Upper arm } \\
\text { Shoulder top }\end{array}$ & $1(3)$ \\
\hline $\begin{array}{l}\text { Upper back } \\
\text { Cpper arm }\end{array}$ & $6(18.2)$ \\
\hline C6/7 & \\
\hline
\end{tabular}

continued for 1 year $(3.2 \pm 1.2)$ ( $p<0.01$ versus preoperative values; Figure $1 \mathrm{~A}$ ). Regarding the significant pain relief assessment, the immediate pain-relieving rate was $78.7 \%$ (26/33), which continued to postoperative 6 months; 1 year later, 22 subjects $(66.6 \%)$ reported that their pain was significantly alleviated (Figure $1 \mathrm{~B}$ ).

According to the Modified MacNab criteria, 63.6$82.1 \%$ considered the surgery "excellent" as pain therapy during the 1-year follow-up period, and 12.1$18.2 \%$ reported that it was "poor" during the study duration (Figure 2). In all studied patients, 9 were treated with additional analgesics after the surgical procedures, while 18 reported soreness at the needle insertion site that completely disappeared in 2 weeks.

No significant complications like hemorrhage, paresthesia, or infection were observed.

\section{Discussion}

In this study, we observed the therapeutic efficacy of coblation annuloplasty in cervical discogenic pain without radiculopathy. The intervention produced significant alleviation of cervical pain that lasted up to 1 year (the entire observation period) after the surgical procedures. Over $60 \%$ of the participants considered the treatment "excellent" at relieving their significant pain.

Table II. Pain characteristics of the patients

\begin{tabular}{|lc|}
\hline Variables & Values \\
\hline Duration of pain [years]: & $4.6 \pm 1.6$ \\
\hline Range (min.-max.) & $0.5-8$ \\
\hline \begin{tabular}{l} 
Distribution of pain, $n$ (\%): \\
\hline Neck base
\end{tabular} & $2(6.1)$ \\
\hline $\begin{array}{l}\text { Neck base/Shoulder top } \\
\text { Neck base/Shoulder top/Upper back }\end{array}$ & $10(30.3)$ \\
\hline \begin{tabular}{l} 
Shoulder top/Upper arm \\
\hline Shoulder top/Upper back/Upper arm
\end{tabular} & $10(30.3)$ \\
\hline \begin{tabular}{l} 
Treated level, $n$ (\%): \\
\hline C4/5
\end{tabular} & $118.2)$ \\
\hline C5/6 & $1(33.3)$ \\
\hline C6/7 & $1(63.6)$ \\
\hline
\end{tabular}


A

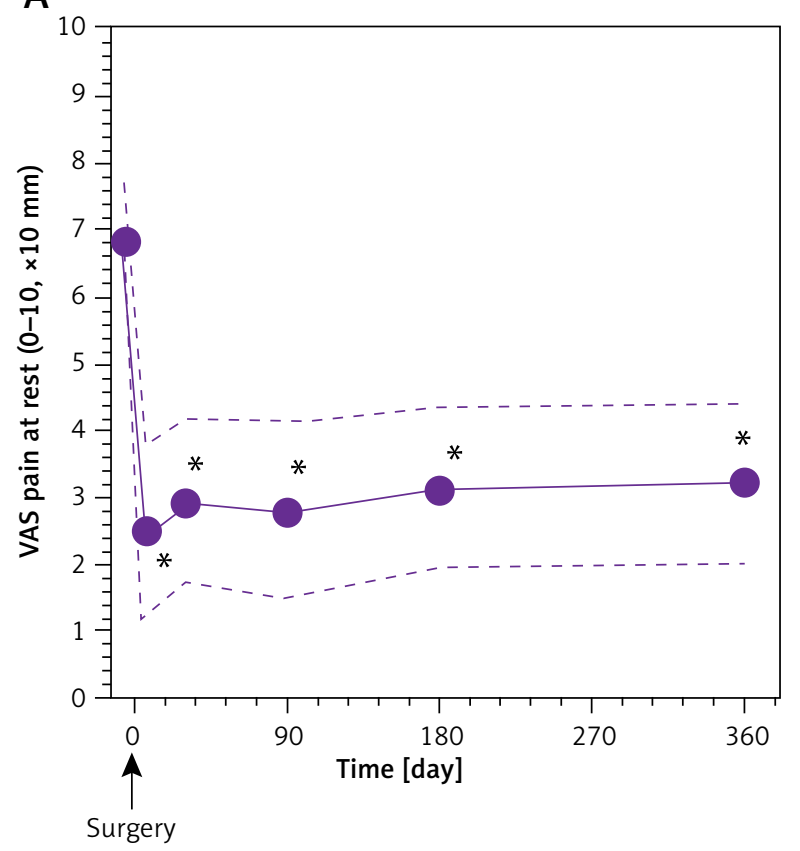

B

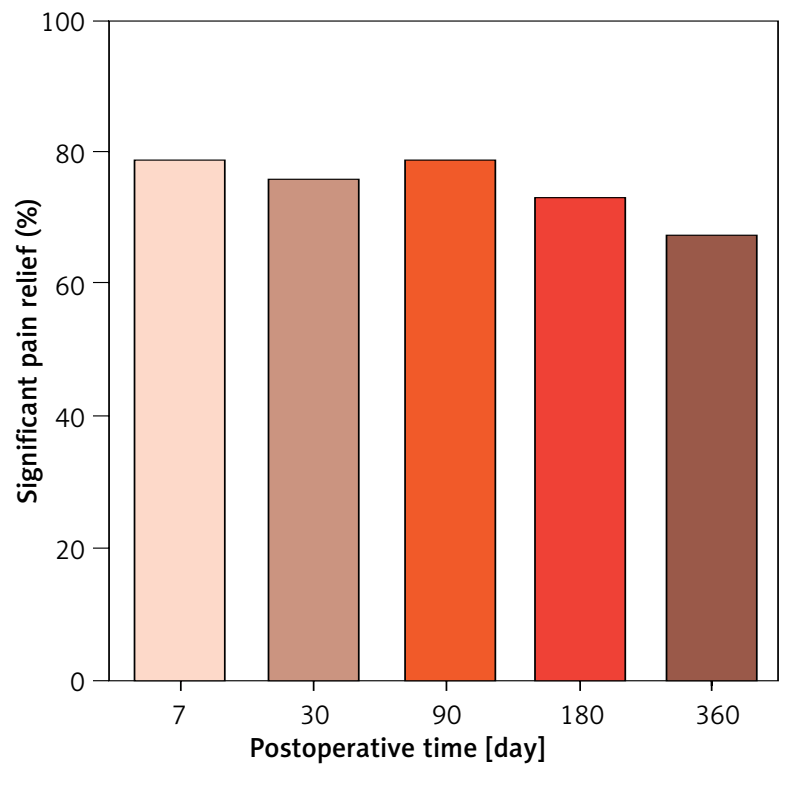

Figure 1. Pain intensity was rated with the VAS gauge at preoperative 1 week and postoperative 1 week and 1, 3, 6 and 12 months; values are shown as mean \pm SD, ${ }^{*} p<0.01$ versus preoperative value (A); significant pain relief rate was observed at postoperative 1 week and 1, 3, 6 and 12 months (B)

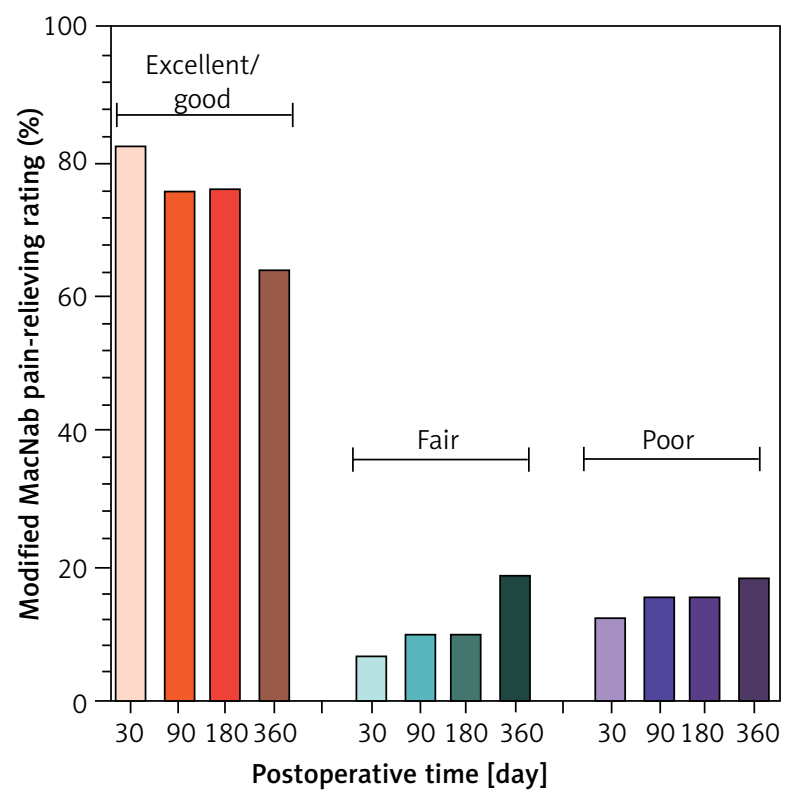

Figure 2. Proportion of patients reporting "excellent", "good", "fair", and "poor" effects at post-operative 1, 3, 6 and 12 months

This study was the first to apply coblation annuloplasty to cervical discogenic pain management. Our original proposal for the application of the tech- nique to cervical discogenic pain was derived with the following considerations: (i) cervical degeneration is the major cause of neck pain [21]; (ii) the outer layers of the annulus fibrosis are normally innervated by interconnected sinuvertebral and vertebral nerves [12, 13]; (iii) chronic degenerative annulopathy causes additional nerve fiber ingrowth into central layers of the annulus fibrosis [20]; and (iv) chronic inflammatory stimulation to the end nerve fibers located in the afflicted disc was the major contributor to the sole discogenic pain without radiculopathy [22]. Thus, the pain-relieving effect of coblation annuloplasty would be as effective as expected if the nerve fibers innervating the annulus fibrosis were ablated, at least in a certain range of time. Our data were in line with this hypothesis, i.e. the neck pain rated with a VAS gauge was substantially attenuated from $\sim 7.0$ to 2.5 , a pain-relieving rate $\left(\triangle V A S / V A S_{\text {origin }}\right)$ of $64 \%$, an effect that lasted up to 1 year, the study observation period.

Regarding coblation annuloplasty, the original notion on this technique was described by Cesaroni and Nardi, who described it as follows: "the active portion of the coblation wand was inserted into the annulus around $5 \mathrm{~mm}$ and make sure that the active 
portion of the wand was deployed into annulus" [23] The primary aim of that study was to evaluate the efficacy of coblation nucleoplasty in treating cervical radicular pain, but their use of coblation nucleoplasty led to coblation annuloplasty. After that, Sim et al. mentioned a procedure in which the active portion of the wand did not pass the imaginary line beyond that connecting the posterior vertebral bodies in the lateral view [24], suggesting that the active portion of the coblation wand was inserted in the posterior annulus. Although both studies did not define the coblation annuloplasty technique, their performance showed a prototype of coblation annuloplasty.

Regarding the total number of participants needed for this study, our Institutional Ethics Examining Committee only approved 40 for this pilot trial to obtain preliminary data on the therapeutic effect of coblation annuloplasty in cervical discogenic pain without radiculopathy; ultimately, 33 patients completed the trial. Although the enrolled number of patients was insufficient to let us draw a confirmative conclusion on the intervention's effect, we believe that our findings were reliable enough for our future studies on this topic. In addition, our study only observed the effect of single coblation annuloplasty on cervical discogenic pain therapy. Thus, we are unsure whether a combination of coblation annuloplasty with other therapeutics such as injection of anti-inflammatory medications would be superior to the sole surgical technique, which requires further investigation, or a randomized controlled trial should be conducted to compare their pain-relieving impact, especially regarding its long-term therapeutic effect.

Complications such as nerve root injury during the procedure were the major concern about its application at the cervical level. In our study, none of our participants showed any signs or symptoms of nerve root injury during or after the intervention. A total of 18 patients reported soreness at the needle insertion site, but it disappeared 2 weeks postoperatively, suggesting that it is not associated with nerve root provocation; besides, it has been described as the most common side effect of coblation technology [25]. Moreover, no complications such as hemorrhage, paresthesia, or infection were observed in our study, as reported by Nardi et al. [26] and Cesaroni and Nardi [27]. Therefore, we concluded that coblation annuloplasty was a safe and minimally invasive technique for managing cervical discogenic pain without radiculopathy.
Some study limitations need to be acknowledged. First, we only followed up all patients for a 1-year period postoperatively, so we cannot claim that the technique was effective at relieving this kind of pain for a much longer duration. Second, the pain duration prior to the surgical procedures was $0.5-8$ years. This wide-ranging pain duration makes the final therapeutic effect vary widely, but we did not perform stratified analyses of the therapeutic effect according to pain duration, so our combined results only reflect an average effect of the technique. That is, we cannot exclude the possible association between different pain durations and the eventual interventional effect. Third, although the VAS pain rating system was the widely used gauge, we cannot eliminate the subjective preference of patients on their own pain ratings. Fourth, the patient's labor type and socioeconomic status were two important factors affecting their perception of pain. We did not perform a sub-analysis of the impacts of these two factors on their final pain rating. Fifth, we cannot exclude the influence of different extents of disc injury prior to the interventional surgery on the final therapeutic effects. Finally, coblation annuloplasty itself is a blind technique, so it is difficult for the physician to completely destroy the nerve endings and remove the inflamed nucleus and annular fissure, which would undoubtedly reduce its therapeutic efficacy.

\section{Conclusions}

Coblation annuloplasty provides dramatic alleviation of neck pain rated with VAS scores and the Modified MacNab score in patients with cervical discogenic pain without radiculopathy. Few significant complications were reported, suggesting that coblation annuloplasty is a feasible and minimally invasive technique for cervical discogenic pain management.

\section{Acknowledgments}

This study was supported by Beijing Municipal Administration of Hospitals Clinical Medicine Development of Special Funding Support (ZYLX201507) and Beijing Municipal Administration of Hospital (PX2019030).

\section{Conflict of interest}

The authors declare no conflict of interest. 


\section{References}

1. Peng B, DePalma MJ. Cervical disc degeneration and neck pain. J Pain Res 2018; 14: 2853-7.

2. Manchikanti L, Abdi S, Atluri S, et al. An update of comprehensive evidence-based guidelines for interventional techniques in chronic spinal pain. Part II: guidance and recommendations. Pain Physician 2016; 16 (2 Suppl): S49-283.

3. Rottenberg Y, Jacobs JM, Stessman J. Prevalence of pain with advancing age brief report. J Am Med Dir Assoc 2015; 16: 264. e1-5.

4. Hoy DG, Bain C, Williams G, et al. A systematic review of the global prevalence of low back pain. Arthritis Rheum 2012; 64: 2028-37.

5. Cote P, Cassidy JD, Carroll L. The Saskatchewan Health and Back Pain Survey. The prevalence of neck pain and related disability in Saskatchewan adults. Spine (Phila Pa 1976) 1998; 23: 1689-98.

6. Cohen SP. Epidemiology, diagnosis, and treatment of neck pain. Mayo Clin Proc 2015; 90: 284-99.

7. Cohen SP, Hooten WM. Advances in the diagnosis and management of neck pain. BMJ 2017; 14: 13221.

8. Benditz A, Brunner M, Zeman F, et al. Effectiveness of a multimodal pain management concept for patients with cervical radiculopathy with focus on cervical epidural injections. Sci Rep 2017; 7: 7866.

9. Manchikanti L, Nampiaparampil DE, Candido KD, et al. Do cervical epidural injections provide long-term relief in neck and upper extremity pain? A systematic review. Pain Physician 2015; 18: 39-60.

10. Moustafa IM, Diab AA. Multimodal treatment program comparing 2 different traction approaches for patients with discogenic cervical radiculopathy: a randomized controlled trial. J Chiropr Med 2014; 13: 157-67.

11. Boswell MV, Trescot AM, Datta S, et al. Interventional techniques: evidence-based practice guidelines in the management of chronic spinal pain. Pain Physician 2007; 10: 7-111.

12. Bogduk N, Windsor M, Inglis A. The innervation of the cervical intervertebral discs. Spine 1988; 13: 2-8.

13. Malinsky J. The ontogenetic development of nerve terminations in the intervertebral discs of man. (Histology of intervertebral discs, $11^{\text {th }}$ communication). Acta Anat 1959; 38: 96-113.

14. Coblation, a cool technology. http://www.arthrocareent.com/ about_coblation_technology. Last access: March 18, 2015.

15. Eggers PE, Thapliyal HV, Matthews LS. Coblation: a newly described method for soft tissue surgery. Res Out Arth Surg 1997; 2:1-4.

16. Groetz SF, Birnbaum K, Meyer C, et al. Thermometry during coblation and radiofrequency ablation of vertebral metastases: a cadaver study. Eur Spine J 2013; 22: 1389-93.

17. Bonaldi G, Baruzzi F, Facchinetti A, et al. Plasma radio-frequency-based diskectomy for treatment of cervical herniated nucleus pulposus: feasibility, safety, and preliminary clinical results. AJNR Am J Neuroradiol 2006; 27: 2104-11.

18. Eichen PM, Achilles N, Konig V, et al. Nucleoplasty, a minimally invasive procedure for disc decompression: a systematic review and meta-analysis of published clinical studies. Pain Physician 2014; 17: e149-73.
19. Gerges FJ, Lipsitz SR, Nedeljkovic SS. A systematic review on the effectiveness of the Nucleoplasty procedure for discogenic pain. Pain Physician 2010; 13: 117-32.

20. Freemont AJ, Peacock TE, Goupille P, et al. Nerve ingrowth into diseased intervertebral disc in chronic back pain. Lancet 1997; 350: 178-81.

21. Oh CH, Kim do Y, Ji GY, et al. Cervical arthroplasty for moderate to severe disc degeneration: clinical and radiological assessments after a minimum follow-up of 18 months - Pfirrmann grade and cervical arthroplasty. Yonsei Med J 2014; 55: 1072-9.

22. Podichetty VK. The aging spine: the role of inflammatory mediators in intervertebral disc degeneration. Cell Mol Biol 2007; 53: 4-18.

23. Cesaroni A, Nardi PV. Plasma disc decompression for contained cervical disc herniation: a randomized, controlled trail. Eur Spine J 2010; 19: 477-86.

24. Sim SE, Ko ES, Kim DK, et al. The results of cervical nucleoplasty in patients with cervical disc disorder: a retrospective clinical study of 22 patients. Korean J Pain 2011; 24: 36-43.

25. Bhagia SM, Slipman CW, Nirschl M, et al. Side effects and complications after percutaneous disc decompression using coblation technology. Am J Phys Med Rehabil 2006; 85: 6-13.

26. Nardi PV, Cabezas D, Cesaroni A. Percutaneous cervical nucleoplasty using coblation technology. Clinical results in fifty consecutive cases. Acta Neurochir Suppl 2005; 92: 73-8.

27. Cesaroni A, Nardi PV. Plasma-mediated disc decompression for contained cervical disc herniation: results through 5 years. Acta Neurochir Suppl 2011; 108: 113-6.

Received: 21.08.2019, accepted: 22.09.2019. 